\title{
Post-Orthodontic Retention: How Much Do People Know and How Do They Decide on Future Orthodontic Treatment and What Do They Expect? A Questionnaire-Based Survey
}

\author{
Sharath Kumar Shetty ${ }^{1 *}$, Mohammed Mazvin Nihal ${ }^{2}$, Vijayananda K. Madhur ${ }^{3}$, Mahesh Kumar $\mathrm{Y}^{4}$
}

\begin{abstract}
${ }^{1}$ Professor \& HOD, Department of Orthodontics \& Dentofacial Orthopaedics , K.V.G Dental College \& Hospital, Sullia, Karnataka, India
${ }^{2}$ Reader, Department of Orthodontics \& Dentofacial Orthopaedics, K.V.G Dental College \& Hospital, Sullia, Karnataka, India

${ }^{3}$ Post Graduate Student, Department of Orthodontics \& Dentofacial Orthopaedics, K.V.G Dental College \& Hospital, Sullia, Karnataka, India

${ }^{4}$ Professor, Department of Orthodontics \& Dentofacial Orthopaedics, K.V.G Dental College \& Hospital, Sullia, Karnataka, India
\end{abstract}

\author{
DOI: $10.36347 /$ sjds.2020.v07i12.003 \\ | Received: 11.12.2020 | Accepted: 21.12.2020 | Published: 24.12.2020
}

*Corresponding author: Sharath Kumar Shetty

Abstract

Original Research Article

Objective: This study aimed to explore the level of knowledge and expectations people deciding on a future orthodontic treatment have about post-orthodontic retention and to investigate the influence of sociocultural characteristics. Material and methods: A total of 134 consecutive people involved as legal decision maker (as patient, parent, or legal guardian) for a prospective orthodontic treatment received before their first appointment a questionnaire to assess their knowledge and expectations about post-orthodontic retention. Data were analysed descriptively, where as predictors were identified with logistic regressions at $P$ value of less than or equal to 0.05 . Results: A total of 134 questionnaires were distributed to prospective orthodontic patients or their parents/legal guardian. The mean age of the 134 participants was $<30$ years $(82.1 \%)$. The majority were male $(70.1$ per cent), of Indian nationality, had a close family member with previous experience of orthodontic treatment (72.4per cent), and reached out for an orthodontic consultation from their own initiative or self motivated (72.9 per cent). The participants' level of knowledge on post-orthodontic retention. half of the participants (82.1 per cent) were aware that retention appliances are used after orthodontic treatment. Finally, the majority believed both that a perfect orthodontic result can guarantee the results' stability (56.7per cent) and that teeth can also move on their own without any orthodontic appliances (63.4 per cent). As far as expectations of the participants toward orthodontic retention are concerned, only 45.5 per cent thought that the retention phase should be less than 1 year, 37.3 per cent thought that it should last between 1 and 3 years, and the remaining 9.7 per cent believed it should extend more than 3-10 years or lifelong. The vast majority of participants (19.4 per cent) rated the stability of the orthodontic results as 'rather important' or 'extremely important', whereas most of them (45.5per cent) preferred a bonded retainer over a removable retention appliance. Most participants considered recalls were needed at 3 months' intervals ( 45.5 per cent) or once a year $(9.7$ per cent). The majority considered that the person most responsible for post-orthodontic stability was the orthodontist, followed by themselves, and finally the general dentist (with percentages being 67.9 per cent). Finally, the vast majority of the participants agreed that it is appropriate to charge fees for recall visits needed during orthodontic retention (74.6 per cent). Conclusions: Although stability of orthodontic treatment results is very important to people deciding about a prospective orthodontic treatment, knowledge regarding the need for post orthodontic retention varies and may at times be contradictious. Sociocultural factors seem to influence the level of knowledge and the expectations on post-orthodontic retention.

Keywords: post-orthodontic retention, self motivated, Orthodontic Treatment, knowledge.

Copyright (C) 2020 The Author(s): This is an open-access article distributed under the terms of the Creative Commons Attribution 4.0 International License (CC BY-NC 4.0) which permits unrestricted use, distribution, and reproduction in any medium for non-commercial use provided the original author and source are credited.

\section{INTRODUCTION}

The significance of retaining tooth alignment after orthodontic treatment to prevent relapse was identified as early as 1904 and its clinical importance has been emphasized since the 1980s-1990s. Although it has become an undisputed fact that orthodontic patients are in need of some type of physical retention of the achieved tooth movement after completion of treatment, orthodontists still debate about the benefits and drawbacks of different retention appliances and protocols [1-9].

The seeming paucity of evidence regarding prospective patients' awareness of post-orthodontic 
retention issues is disturbing. The necessity to understand the expectations and assess the level of knowledge of people interested in an orthodontic treatment is accentuated by the fact that postorthodontic satisfaction is strongly related to the patients' perception of tooth stability and responsibilities during the retention phase [10-16].

The primary aim of this study was, therefore, to assess the level of knowledge and the expectations concerning post-orthodontic retention of people enrolled for an orthodontic treatment, before their first orthodontic appointment or assessment. The secondary aim was to discern whether demographical and sociocultural characteristics of the participants influence their level of knowledge and their expectations [17-20].

\section{MATERIAL AND METHODS \\ Study Design and Participants}

A self-developed online questionnaire was distributed to patients more than 16 years of age. All participants were briefed about the goal of this survey, its voluntary basis, and its anonymized design. The participants were instructed to fill out the questionnaire silently and independently, without time restriction.

The questionnaire consisted of three sections of closed-ended Queries1. items relating to participant demographical and sociocultural characteristics, 2. items concerning the participant's knowledge about post-orthodontic retention need, and 3. Items focusing on the participant's attitude and expectations toward orthodontic retention.

The questionnaire was distributed to all consecutive patients/ decision makers who were about to have their first orthodontic appointment and who had agreed to participate.

\section{Statistical Analysis}

Descriptive statistics were calculated for all variables, including means and standard deviations for continuous variables or absolute and relative frequencies for categorical variables. Binary logistic regressions were performed, after checking for possible violations of assumptions, to identify predictors for the response to a handful of selected questions of interest to the patient and/or the orthodontist. Each independent variable was initially added in a univariable model with the question response as dependent variable and all collected participant demographical/ sociocultural characteristics as independent variables. Subsequently, a multivariable model was built and all independent variables with $\mathrm{P}$ value of less than or equal to 0.20 from the first model were added to account for confounders.

\section{Patients Responses \\ Demographic Data}

1) Gender

134 responses

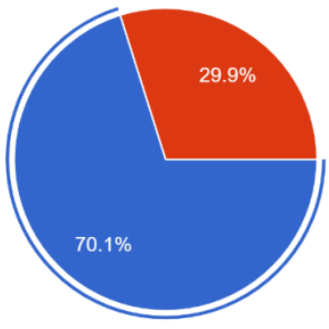

Male

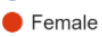

2) State

128 responses

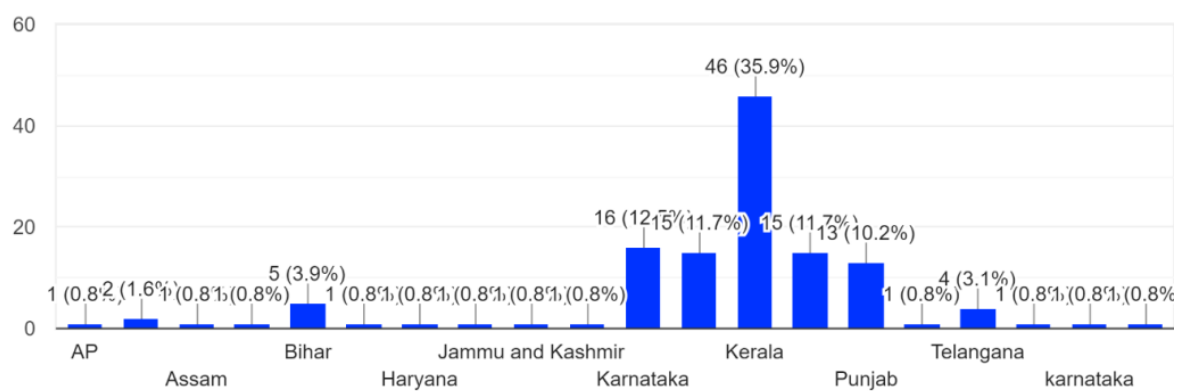


3) Age

134 responses

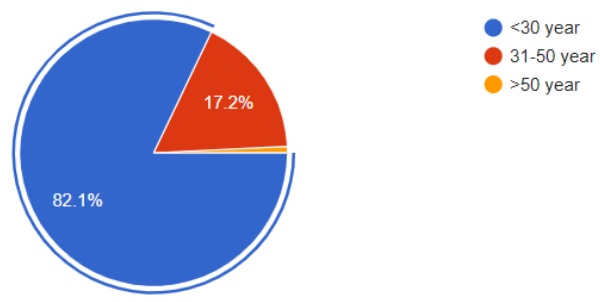

4) Reason of consultation

133 responses

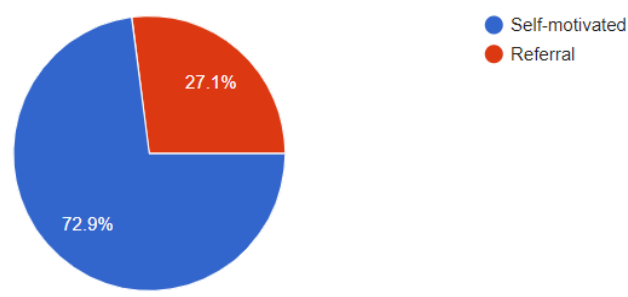

5) Previous experiences with orthodontics within close family

134 responses

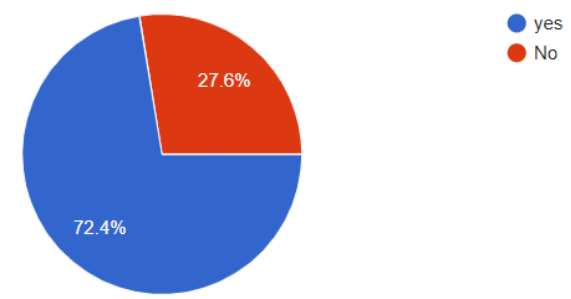

6) Clinic

132 responses

\section{Participants' level of knowledge}

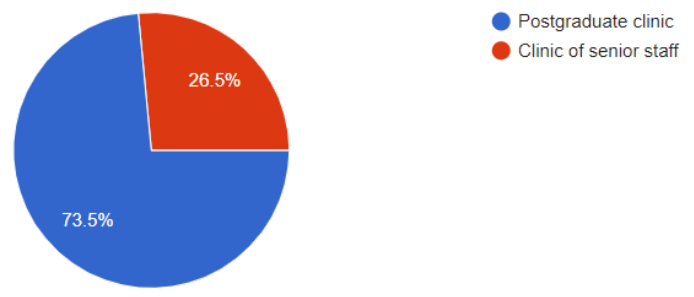

7) Are you aware that appliances are used for retention after orthodontic treatment? 134 responses

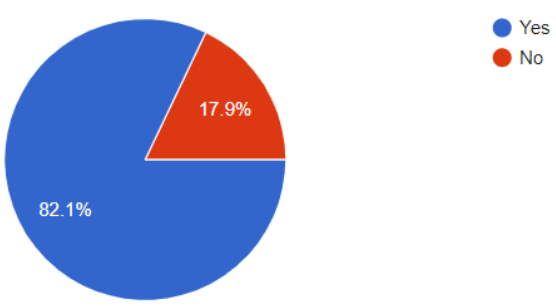


8) Do you believe a perfect treatment result can guarantee stability?

134 responses

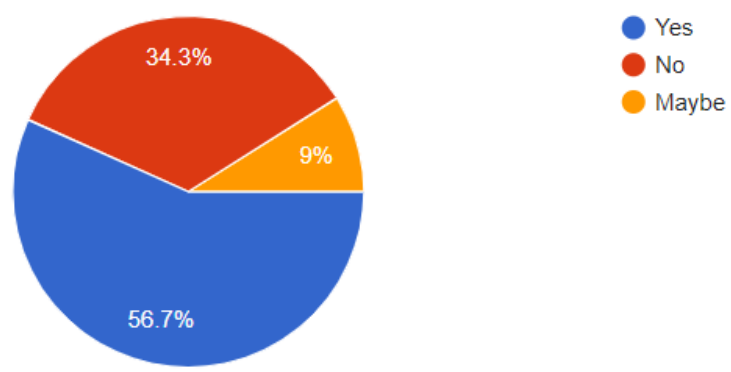

9) Do you think that teeth can also move without orthodontic appliances? 134 responses

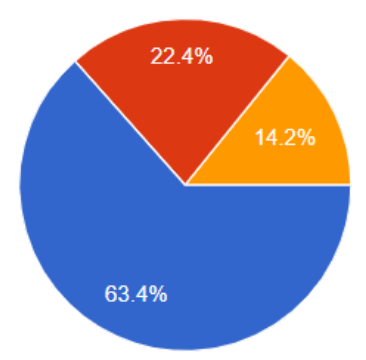

Yes

No

Maybe

Participants' expectations in orthodontic retention

10) How long do you think should the retention phase be?

134 responses

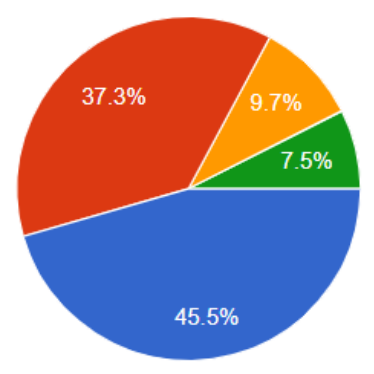

$<1$ year

1-3 years

3-10 years

Lifelong

11) How important is a stable result for you

134 responses

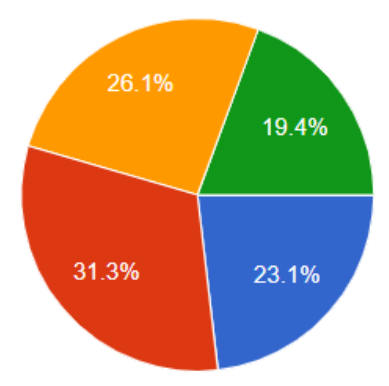

Not important

Ambivalent

Rather important

Extremely important 
12) Which type of retention device would you favour

134 responses

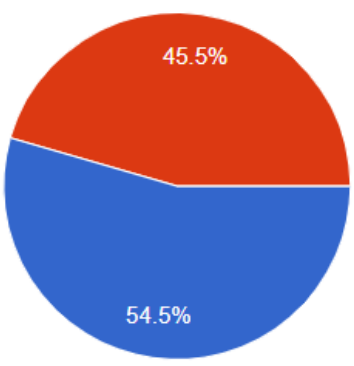

Removable device

Bonded device

13) At which interval do you believe is a recall necessary

134 responses

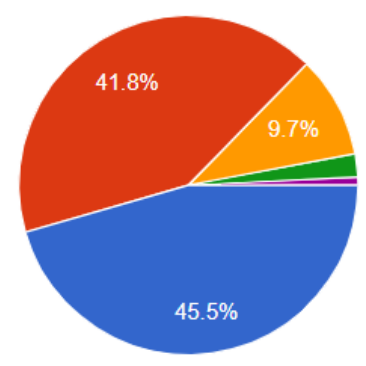

Every 3 month

Every 6 month

Yearly

Every 2nd year

Every 5 th year

14) Who do you consider responsible for the stability after orthodontictreatment?

134 responses

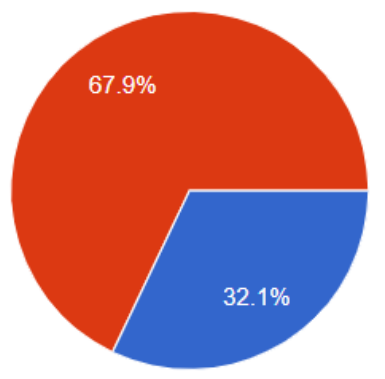

General dentist

Orthodontist

15) Do you think it is appropriate to charge for recall visits

134 responses

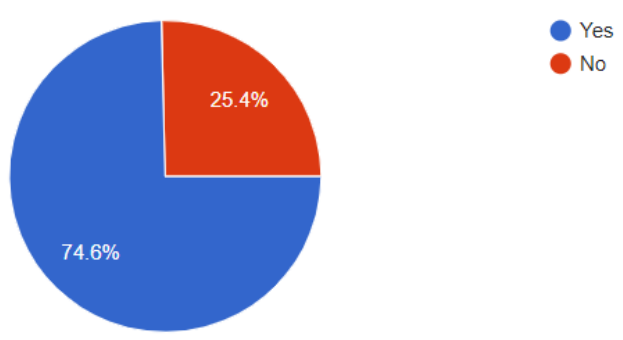




\section{RESULTS}

A total of 134 questionnaires were distributed to prospective orthodontic patients or their parents/legal guardian. The mean age of the 134 participants was $<30$ years $(82.1 \%)$. The majority were male (70.1 per cent), of Indian nationality, had a close family member with previous experience of orthodontic treatment (72.4per cent), and reached out for an orthodontic consultation from their own initiative or self motivated (72.9 per cent). The participants' level of knowledge on postorthodontic retention. half of the participants (82.1 per cent) were aware that retention appliances are used after orthodontic treatment. Finally, the majority believed both that a perfect orthodontic result can guarantee the results' stability (56.7per cent) and that teeth can also move on their own without any orthodontic appliances (63.4 per cent).

As far as expectations of the participants toward orthodontic retention are concerned, only 45.5 per cent thought that the retention phase should be less than 1 year, 37.3per cent thought that it should last between 1 and 3 years, and the remaining 9.7 per cent believed it should extend more than 3-10 years or lifelong. The vast majority of participants (19.4 per cent) rated the stability of the orthodontic results as 'rather important' or 'extremely important', whereas most of them (45.5per cent) preferred a bonded retainer over a removable retention appliance. Most participants considered recalls were needed at 3 months' intervals (45.5 per cent) or once a year (9.7 per cent). The majority considered that the person most responsible for post-orthodontic stability was the orthodontist, followed by themselves, and finally the general dentist (with percentages being 67.9 per cent). Finally, the vast majority of the participants agreed that it is appropriate to charge fees for recall visits needed during orthodontic retention (74.6 per cent).

\section{DISCUSSION}

This study seems to be the first attempt to empirically address individuals before any orthodontic appointment in order to assess the level of knowledge and the expectations of people deciding about a prospective orthodontic treatment regarding postorthodontic retention. We considered it to be of high interest to target people who are in charge of deciding about an impending orthodontic treatment involving a prolonged retention protocol, and to identify what they know and what they expect-precisely at the time of their consent to the forthcoming treatment. Although the number of participants and the return rate in this investigation can be considered sufficient to allow statistical tests beyond simple descriptive statistics, it was decided to refrain from a hypothesis-driven approach and remain entirely observational [20-31].

\section{CONCLUSION}

This questionnaire-based survey reveals that guaranteeing the result of orthodontic treatment is of great importance to people deciding about a prospective orthodontic treatment. Knowledge regarding possible post-orthodontic relapse and the need for postorthodontic retention varies and seems to be influenced by sociocultural factors. A certain consensus amongst Swiss prospective orthodontic patients/decision makers seems to exist regarding responsibility, necessity of recalls, the takeover of costs, and preferences in retainer devices. On the basis of the results of this survey, it appears mandatory to adequately inform prospective orthodontic patients and their parents/guardians about the need of post-orthodontic retention, and the extent of future commitment expected from them during the retention phase.

\section{REFERENCES}

1. Kaplan H. The logic of modern retention procedures. American Journal of Orthodontics and Dentofacial Orthopedics. 1988 Apr 1;93(4):32540.

2. Little RM. Stability and relapse of dental arch alignment. British journal of orthodontics. 1990 Aug;17(3):235-41.

3. Little RM, Riedel RA, Artun J. An evaluation of changes in mandibular anterior alignment from 10 to 20 years postretention. American Journal of Orthodontics and Dentofacial Orthopedics. 1988 May 1;93(5):423-8.

4. Blake M, Bibby K. Retention and stability: a review of the literature. American Journal of Orthodontics and Dentofacial Orthopedics. 1998 Sep 1;114(3):299-306.

5. Andriekute A, Vasiliauskas A, Sidlauskas A. A survey of protocols and trends in orthodontic retention. Progress in orthodontics. 2017 Dec $1 ; 18(1): 31$.

6. Littlewood SJ, Millett DT, Doubleday B, Bearn DR, Worthington HV. Retention procedures for stabilising tooth position after treatment with orthodontic braces. Cochrane Database of Systematic Reviews. 2016(1).

7. Wong PM, Freer TJ. A comprehensive survey of retention procedures in Australia and New Zealand. Australian orthodontic journal. 2004 Nov;20(2):99-106.

8. Meade MJ, Millett D. Retention protocols and use of vacuum-formed retainers among specialist orthodontists. Journal of orthodontics. 2013 Dec;40(4):318-25.

9. Ab Rahman N, Low TF, Idris NS. A survey on retention practice among orthodontists in Malaysia. The Korean Journal of Orthodontics. 2016 Jan 1;46(1):36-41.

10. Renkema AM, Hélène Sips ET, Bronkhorst E, Kuijpers-Jagtman AM. A survey on orthodontic retention procedures in The Netherlands. The 
European Journal of Orthodontics. 2009 Aug 1;31(4):432-7.

11. Padmos JA, Fudalej PS, Renkema AM. Epidemiologic study of orthodontic retention procedures. American journal of orthodontics and dentofacial orthopedics. 2018 Apr 1;153(4):496504.

12. Al-Jewair TS, Hamidaddin MA, Alotaibi HM, Alqahtani ND, Albarakati SF, Alkofide EA, AlMoammar KA. Retention practices and factors affecting retainer choice among orthodontists in Saudi Arabia. Saudi Medical Journal. 2016 Aug;37(8):895-901.

13. Arnold SN, Pandis N, Patcas R. Factors influencing fixed retention practices in Germanspeaking Switzerland. Journal of Orofacial Orthopedics/Fortschritte der Kieferorthopädie. 2014 Nov 1;75(6):446-58.

14. Habegger M, Renkema AM, Bronkhorst E, Fudalej PS, Katsaros C. A survey of general dentists regarding orthodontic retention procedures. European journal of orthodontics. 2017 Feb 1;39(1):69-75.

15. Lai Heuberger C, Grossen J, Renkema AM, Bronkhorst E, Fudalej P, Katsaros C. Orthodontic retention procedures in Switzerland. Swiss dental journal. 2014;124(6):655-61.

16. Singh P, Grammati S, Kirschen R. Orthodontic retention patterns in the United Kingdom. Journal of orthodontics. 2009 Jun;36(2):115-21.

17. Bibona K, Shroff B, Best AM, Lindauer SJ. Factors affecting orthodontists' management of the retention phase. The Angle Orthodontist. 2014 Mar 1;84(2):225-30.

18. Valiathan M, Hughes E. Results of a survey-based study to identify common retention practices in the United States. American Journal of Orthodontics and Dentofacial Orthopedics. 2010 Feb 1;137(2):170-7.

19. Pratt MC, Kluemper GT, Hartsfield Jr JK, Fardo D, Nash DA. Evaluation of retention protocols among members of the American Association of Orthodontists in the United States. American Journal of Orthodontics and Dentofacial Orthopedics. 2011 Oct 1;140(4):520-6.

20. Vandevska-Radunovic V, Espeland L, Stenvik A. Retention: Type, duration and need for common guidelines. A survey of Norwegian orthodontists. ORTHODONTICS: The Art \& Practice of Dentofacial Enhancement. 2013 Mar 1;14(1):e110-e117.
21. Yao J, Li DD, Yang YQ, McGrath CP, Mattheos $\mathrm{N}$. What are patients' expectations of orthodontic treatment: a systematic review. BMC oral health. 2016 Dec;16(1):1-8.

22. Mollov ND, Lindauer SJ, Best AM, Shroff B, Tufekci E. Patient attitudes toward retention and perceptions of treatment success. The Angle Orthodontist. $2010 \mathrm{Jul} ; 80(4)$ :656-61.

23. Swiss Academy of Medical Sciences. Assessment of capacity in medical practice. In Medical-Ethical Guidelines. Berne, Switzerland, 2018; 20. https://www.samw.ch/dam/jcr:68d1793c-63434e84-bed0-

c1532928e42e/guidelines_sams_assessment_of_ca pacity.pdf (5 April 2019, date last accessed).

24. Papageorgiou $\mathrm{SN}$, Eliades $\mathrm{T}$, Hämmerle $\mathrm{CH}$. Frequency of infraposition and missing contact points in implant- supported restorations within natural dentitions over time: A systematic review with meta- analysis. Clinical Oral Implants Research. 2018 Oct;29:309-25.

25. Thilander B, Ödman J, Lekholm U. Orthodontic aspects of the use of oral implants in adolescents: a 10- year follow- up study. European journal of orthodontics. 2001 Dec 1;23(6):715-31.

26. Henrikson J, Persson M, Thilander B. Long-term stability of dental arch form in normal occlusion from 13 to 31 years of age. The European Journal of Orthodontics. 2001 Feb 1;23(1):51-61.

27. Thilander B. Dentoalveolar development in subjects with normal occlusion. A longitudinal study between the ages of 5 and 31 years. The European Journal of Orthodontics. 2009 Apr 1;31(2):109-20.

28. Kahl-Nieke B, Fischbach H, Schwarze CW. Postretention crowding and incisor irregularity: a longterm follow-up evaluation of stability and relapse. British Journal of Orthodontics. 1995 Aug;22(3):249-57.

29. Little RM. Stability and relapse of mandibular anterior alignment: University of Washington studies. InSeminars in orthodontics 1999 Sep 1 (Vol. 5, No. 3, pp. 191-204). WB Saunders.

30. Pavel MS, Chakrabarty S, Gow J. Assessing willingness to pay for health care quality improvements. BMC health services research. 2015 Dec 1;15(1):43

31. Furnham A, Von Stumm S, Fenton-O’Creevy M. Sex differences in money pathology in the general population. Social indicators research. $2015 \mathrm{Sep}$ 1;123(3):701-11. 